\title{
Compact hadron driver for cancer therapies using continuous energy sweep scanning
}

\author{
Leo Kwee Wah, ${ }^{1}$ Takumi Monma, ${ }^{2,3}$ Toshikazu Adachi, ${ }^{3,4}$ Tadamichi Kawakubo, ${ }^{3}$ \\ Tanuja Dixit, ${ }^{5}$ and Ken Takayama ${ }^{2,3,4,6}$ \\ ${ }^{1}$ Malaysian Nuclear Agency, Kajang, Bangi 43000, Malaysia \\ ${ }^{2}$ Tokyo Institute of Technology, Nagatsuda, Kanagawa 226-8503, Japan \\ ${ }^{3}$ High Energy Accelerator Research Organization (KEK), Tsukuba, Ibaraki 305-0801, Japan \\ ${ }^{4}$ The Graduate University for Advanced Studies (SOKENDAI), Hayama, Kanagawa 240-0193, Japan \\ ${ }^{5}$ Society for Applied Microwave Electronics Engineering \& Research (SAMEER), Mumbai 400076, India \\ ${ }^{6}$ Tokyo City University, Setagaya, Tokyo 158-8557, Japan
}

(Received 14 July 2015; published 26 April 2016)

\begin{abstract}
A design of a compact hadron driver for future cancer therapies based on the induction synchrotron concept is presented. To realize a slow extraction technique in a fast-cycling synchrotron, which allows energy sweep beam scanning, a zero momentum-dispersion $D(s)$ region and a high flat $D(s)$ region are necessary. The proposed design meets both requirements. The lattice has two-fold symmetry with a circumference of $52.8 \mathrm{~m}$, a 2-m dispersion-free straight section, and a 3-m-long large flat dispersion straight section. Assuming a 1.5-T bending magnet, the ring can deliver heavy ions $(200 \mathrm{MeV} / \mathrm{u})$ at $10 \mathrm{~Hz}$. A beam fraction is dropped from the barrier bucket at the desired timing, and the increasing negative momentum deviation of this beam fraction becomes large enough for the fraction to fall in the electrostatic septum extraction gap, which is placed at the large $D(s)$ region. The programmed energy sweep extraction enables scanning beam irradiation on a cancer site in depth without an energy degrader, avoiding the production of secondary particles and the degradation of emittance. Details of the lattice parameters and computer simulations for slow extraction are discussed. An example extraction scenario is presented. Qualities of the spilled beam such as emittance and momentum spread are discussed, as well as necessary functions and parameters required for the extraction system.
\end{abstract}

DOI: 10.1103/PhysRevAccelBeams.19.042802

\section{INTRODUCTION}

Nowadays, 3D spot scanning of hadron beams applied to cancer tissues in human organs is of great concern in society. Here we focus on spot scanning in depth. Several ways to realize such spot scanning are known and have been demonstrated. One of them is the well-known technique of using a range shifter. In this technique, the hadron beam is extracted with the same energy and the before-irradiation beam energy is changed by using a combination of plastic plates with different thicknesses, at the expense of producing a huge number of secondary particles created by interactions with the range shifter material, resulting in large residual radiation and degraded transverse emittance. Accuracy in the z-direction is determined by mechanical control of the range shifter. Techniques based on a rotating range shifter with a taper may be classified into this category. In contrast, a technique of changing the extraction beam energy itself has been demonstrated in the Hitachi slow-cycling proton

Published by the American Physical Society under the terms of the Creative Commons Attribution 3.0 License. Further distribution of this work must maintain attribution to the author(s) and the published article's title, journal citation, and DOI. synchrotron, where the excitation ramping pattern of guiding magnets is varied by using a different acceleration cycle and the beam is continually and slowly extracted on the flat top by a combination of third-integer resonance and rf deflection. Respiration gating operation is realized by real-time feedback of human body information to the rf deflection device in the Hitachi system [1]. Unfortunately, the change in the ramping pattern is not continuous but discrete, changing pulse by pulse. The smoothness of spot scanning in the z-direction in the demonstrated example is intrinsically limited by this characteristic. Continuous irradiation of moving organs, which requires energy sweeps of a few tens of milliseconds, has not been realized yet. If continuous and smoothed scanning in depth via irradiation in cancer therapies is implemented, the flexibility of treatment should extensively expand.

In principle, continuous extraction from a fast-cycling synchrotron on the way to the end of acceleration may be possible by using half-integer or third-integer resonances, although this has not yet been demonstrated. Any rfmanipulating extraction method in combination with a change in the equilibrium orbit may also be possible. Recently, the idea of energy sweep extraction in a single acceleration cycle from a fast-cycling synchrotron has been proposed in the iRCMS project [2]. This is depicted in Fig. 1, although the details are not open to the public. 


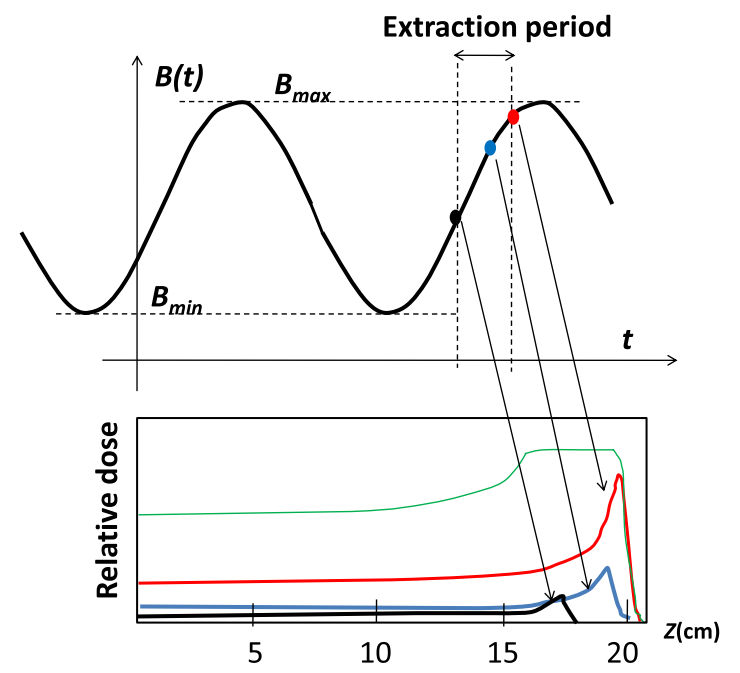

FIG. 1. Energy sweep extraction in one acceleration cycle and the integrated dose along the path, where $B(t)$ is the magnetic flux density of the guiding magnet.

In this paper, a practical method to realize energy sweep extraction from a fast-cycling synchrotron is proposed. We assume a fast-cycling induction synchrotron [3]. A hadron bunch captured in the barrier bucket is continuously accelerated by the induction flat voltage, and a fraction of the beam bunch is spilled out from the stable barrier bucket by nonadiabatically changing the timing of the acceleration voltage controlling trigger signal in the desired time period. Equilibrium orbits of spilled-out particles move inward depending on the dispersion function $D(s)$, and those particles enter into the electrostatic septum gap region to be further deflected inward, and then propagate through the extraction region downstream consisting of extraction devices such as a septum magnet or Lambertson magnet installed on the extraction beam line. The start of the extraction and the number of spilled-out particles are simply determined by controlling the gate signal. Thus, we can obtain a driver beam for cancer therapy with 3D spot scanning, the energy of which changes continuously in a single acceleration cycle.

The organization of this paper is as follows. In Sec. II, more details of the idea of the in-beam energy sweep extraction will be given. A ring lattice design that allows the energy sweep extraction is discussed in the next section. Section IV will be devoted to 3D beam tracking simulations, which are used to confirm the idea's feasibility. In Sec. V, crucial hardware, such as the electrostatic septum and Lambertson magnet downstream, which must work for a beam with temporally varying energy, will be discussed, including guiding magnet components. In Sec. VI, beam loss associated with the energy sweep extraction, which is caused by small-angle multiple scattering due to the thin septum wires material, is estimated. Finally, we summarize the paper.

\section{IDEA OF IN-BEAM ENERGY SWEEP EXTRACTION AND SPOT SCANNING}

An ideal spot scanning system would allow 3D scanning on a cancer site by a pencil beam with precise accuracy, as shown in Fig. 2. If the cancer site in a moving organ is identified by a technique such as single-photon emission computed tomography [4] with improved spatial resolution and the beam deposit spot is identified by means of in-beam positron emission tomography (PET; C-11) [5] in real time, then continuous irradiation of a moving organ may become possible. In the future, a gigantic and extremely expensive gantry or respiratory-synchronized irradiation may become unnecessary.

The technology for the above purpose is not yet sufficiently matured. In this sense, there are many things that need to be developed in the medical physics field. For instance, the recent report from the Workshop on Ion Beam Therapy (Jan. 9-11, 2013) [6] summarizes crucial tasks that we have to do from now in order to realize an ideal ion beam facility. Determining how a pencil beam is deposited at a desired position may be an accelerator-side issue, especially for moving positions with precise accuracy and dose uniformity. To meet this requirement, moving range shifters have been used since the very early days of development. As a next approach, extraction beam energy itself has been varied by changing the ramping pattern of the guiding magnets. Range shifters are accompanied by production of a large number of secondary particles, resulting in an increase of the residual radiation level and some degradation of transverse beam emittance. Varying the energy of extraction beams still has some limitations in terms of continuous change in energy, as mentioned earlier. In addition, this type of operation seems undesirable from the perspective of steady-state operation by a driver machine, although this can be further improved.

Here, another approach to continuously changing the beam energy in line with the demands for 3D spot scanning is proposed. The basic idea is quite simple. A fast-cycling induction synchrotron is assumed as the driver machine. All guiding magnets, including the guiding magnets on the extraction beam line, are always operated in an identical pattern. A hadron bunch captured in the barrier bucket, which is created with a combination of trapezoidal voltage

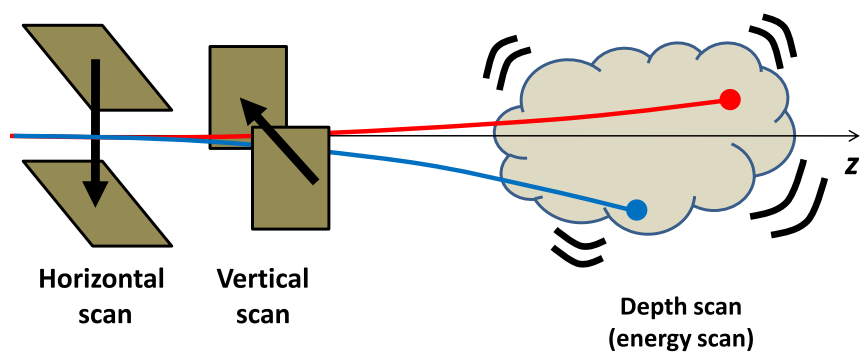

FIG. 2. 3D Schematic of a cancer site and scanning spots. 


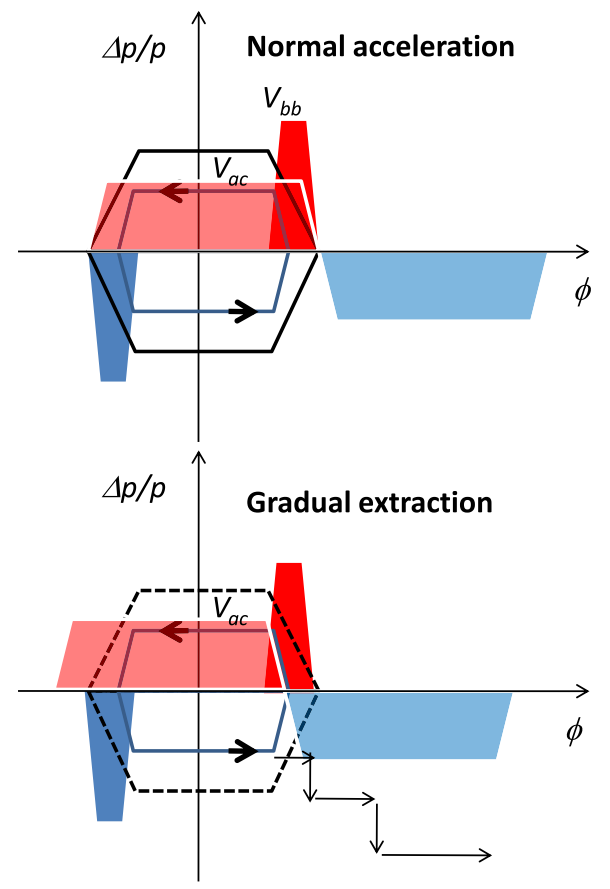

FIG. 3. Barrier bucket and acceleration voltage pulse with its reset voltage pulse (upper). Tracks of particles that have left the trapping region encircled by a broken line (lower).

pulses of opposite polarity, is accelerated with a long flat voltage pulse. All voltage pulses are generated at the induction acceleration cells, which are energized by a switching power supply employing solid-state switching elements such as $\mathrm{SiC}$ devices [7]. Since the solid-state switches are triggered by the gate signal, the necessary voltage pulses can be generated with the desired timing. Consequently, annihilation and creation of both voltage pulses can be realized with perfect freedom. When the falling edge of an accelerating voltage pulse is moved to left in phase, as seen in Fig. 3, the particles there repeatedly experience a shortage in the accelerating voltage. Eventually, the particles leaving the barrier region simply drift on the time or phase axis, depending on the increasing negative momentum deviation $\Delta p / p$. The last phase motion of particles, having left the trapped region, is depicted in Fig. 3. The particles achieve no effective acceleration voltage hereafter because they are affected by the set and reset voltages of the same amplitude. They move further downward in the momentum phase space $(\phi$, $\Delta p / p$ ). Thus a beam spill leaking from the barrier bucket is produced in the required way. Necessarily, their equilibrium orbits change inward because of positive $D(s)$ in most cases. Those particles eventually enter into the electrostatic septum region, which is placed in the dispersion region with the largest flat momentum. Electric fields of order $20 \mathrm{kV} / \mathrm{cm}$ further deflect particles inward at a sharper angle. The extraction beam line is connected to the downstream extraction region consisting of the septum magnets or Lambertson magnet. The essential features of the idea are shown in Fig. 4.

It is crucial that the time period for extraction and the spill intensity are manipulated by controlling the gate signal. This gate signal control is carried out by using a combination of a field-programmable gate array and a PC, in which necessary data (depending on patient or cancer site) are input in advance. This is an important part of the treatment protocol.

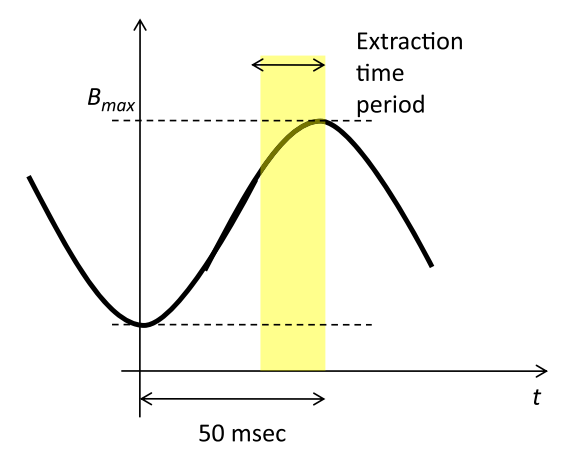

Extraction method:

This relies on a finite momentum dispersion function and large momentum deviation

$\mathrm{x}=\mathrm{D}(\mathrm{s}) \Delta \mathrm{p} / \mathrm{p}$

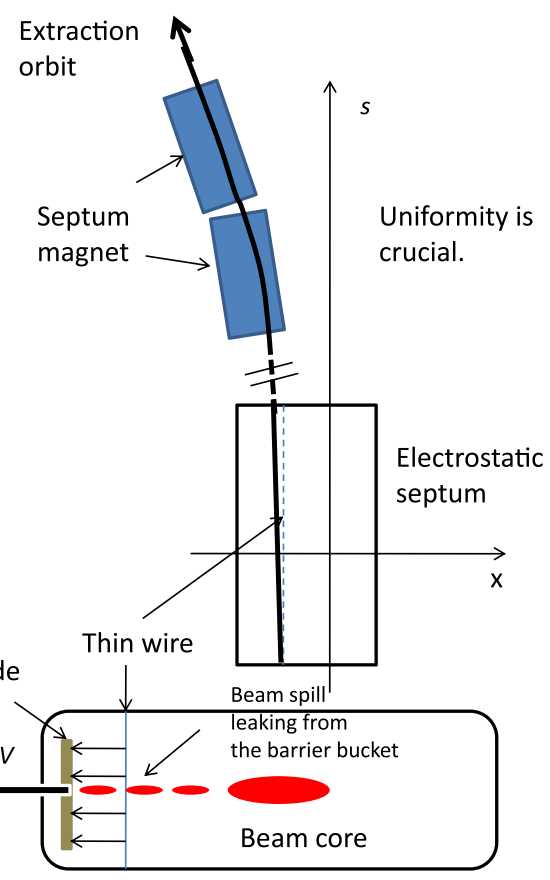

FIG. 4. Essential feature of energy sweep extraction. 


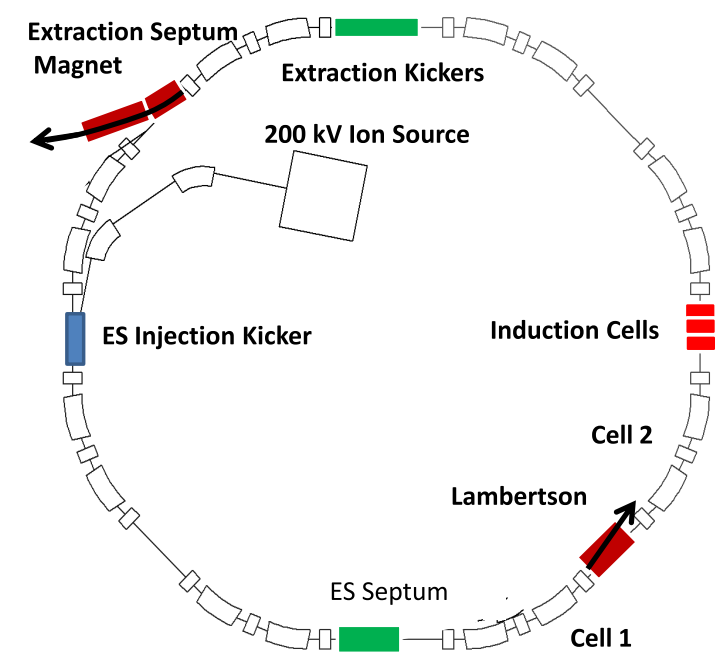

FIG. 5. Overview of the driver ring.

To realize this scheme, we pose specific demands on the machine lattice. They are summarized as follows. (1) Dispersion-free region for induction acceleration devices and injection devices (2) Localized large flat dispersion region with a length of $3 \mathrm{~m}$ (3) Lattice to accommodate the fast extraction region with a desired phase advance.

\section{LATTICE DESIGN WITH LARGE, FLAT DISPERSION FUNCTION AND DISPERSION-FREE SECTION}

The beam energy for the cancer therapy driver is chosen from within $50-200 \mathrm{MeV} / \mathrm{u}$ for protons and for $\mathrm{C}^{6+}$. Assuming the use of normal conducting bending magnets with a maximum flux density of $1.5 \mathrm{~T}$, the circumference of a driver ring is about $50-60 \mathrm{~m}$. This ring must be equipped with an injection device, an extraction device, induction acceleration cells, steering magnets, a chromaticity correction system, and various beam diagnosis systems, as well as synchrotrons for other purposes. The main purpose of this section is not to give a full lattice design but to describe the reference design, which has the essential function to realize energy sweep scanning.

The ring has two-fold symmetry as shown in Fig. 5. Its superlattice has mirror symmetry, which consists of two sorts of F-B-D-B-F cells in Fig. 6. The lattice profile is similar to that of the design by the PIMMS group at CERN [8] Two long dispersion-free straight sections are occupied by the injection system and induction acceleration cells for acceleration and confinement. One long straight section with a large flat dispersion function is occupied by the extraction system for energy sweep scanning. The other is partially occupied by the kicker magnets for fast extraction, which is used in combination with the septum magnet placed downstream with a betatron phase advance of 90 degrees.

The reference design does not employ a large-scale injector such as an RFQ or hadron linac, as well as the existing induction synchrotron [3]. The ion source is embedded in the 200-kV high-voltage platform (HVP). A laser ablation ion source [9] is the most promising candidate for fully stripped carbon ions. A hadron beam, which is extracted from the HVP and propagates through the minimum beam transport line, is immediately injected in one turn by the electrostatic injection kicker, which is turned off by a matrix array of solid-state switching elements instead of the usual thyratron vacuum tube [10].

The machine and beam parameters are listed in Table I. The lattice function for the super-periodicity is given in Fig. 7. The lattice component parameters are also given in Table II.

\section{ENERGY SWEEP EXTRACTION SIMULATIONS}

In this driver ring, a hadron bunch trapped in the barrier bucket is accelerated with the induction voltage pulse. The essential features of the acceleration and confinement are the same as those of the existing induction synchrotron, as mentioned earlier. Here, particle tracking simulations in the longitudinal phase space are described, where macroparticles injected into the ring are tracked until the end of acceleration. When the time for extraction arrives, the flat edge of the $V_{\text {acc }}$ pulse is moved to the rising edge of the positive $V_{b b}$ pulse in one turn. Insufficient magnitude of the acceleration voltage allows an artificial and continuous leak of a fraction of macroparticles beyond the assumed extraction timing.

\section{A. Acceleration from injection to extraction timing}

The essential features of the motion of macroparticles in the longitudinal phase space are the same as those in a fastcycling induction synchrotron, such as the KEK digital accelerator, at least up to the extraction timing. Temporal evolutions of the guiding magnetic flux, energy per

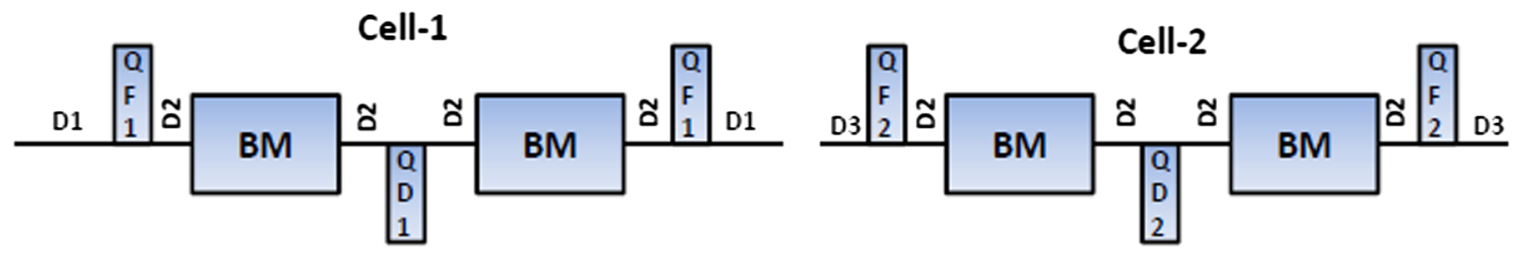

FIG. 6. Cell structures. 
TABLE I. Machine and beam parameters.

\begin{tabular}{ll}
\hline \hline Energy & $656 \mathrm{MeV}$ for proton $200 \mathrm{MeV} /$ nucleon for \\
& $A / Q=2$ ion \\
$C_{0}$ & $52.8 \mathrm{~m}$ \\
Ion species & Gaseous/metal ions \\
Ion source & Laser ablation IS ECRIS \\
Injector & $200 \mathrm{kV}$ (electrostatic) \\
Ring & Fast cycling $(10 \mathrm{~Hz})$ \\
& $B_{\max }=1.5 \mathrm{~T}$ \\
& $\rho=2.8662 \mathrm{~m}$ \\
& FODOF cell with edge focus of B \\
& Mirror symmetry \\
& $\nu_{x} / \nu_{y}=1.3143 / 1.4635$ \\
& 2 m long dispersion-free region $3 \mathrm{~m} \mathrm{long}$ \\
& flat large dispersion region \\
& $a_{p}=0.273088 \gamma_{T}=1.92, E_{T}=$ \\
& $864.7 \mathrm{MeV}$ \\
Acceleration & Induction cells driven by SPS employing \\
& SiC-MOSFET $V_{\text {acc }}=\rho C_{0} d B / d t$ \\
& $($ max $7 \mathrm{kV})$ \\
Vacuum & $10^{-8}$ Pa \\
\hline \hline
\end{tabular}

nucleon, revolution period, and required acceleration voltage per turn over the entire acceleration cycle are shown in Fig. 8. It is assumed that $V_{a c}$ of the ideal required acceleration voltage $\left(V_{a c c}=\rho C_{0} d B / d t\right)$ is always provided in the simulations. In addition, $V_{b b}$ is fixed to be $0.2 \mathrm{kV}$ through the acceleration cycle.

A rectangular uniform macroparticle distribution with a half-momentum deviation of $5 \times 10^{-3}$ and a pulse length of $4 \mu \mathrm{s}$ is assumed in the longitudinal phase space $(\phi, \Delta p / p)$ at the injection timing. All macroparticles are tracked by the induction acceleration step equations, which are written as

$$
\left\{\begin{array}{l}
E_{n+1}=E_{n}+Q e \cdot\left[V_{a c}\left(\phi_{n}\right)+V_{b b}\left(\phi_{n}\right)\right] \\
\phi_{n+1}=\phi_{n}+\frac{2 \pi \cdot \eta}{\left(\beta_{n+1}^{s}\right)^{2} E_{n+1}^{s}} \cdot(\Delta E)_{n+1}
\end{array}\right.
$$

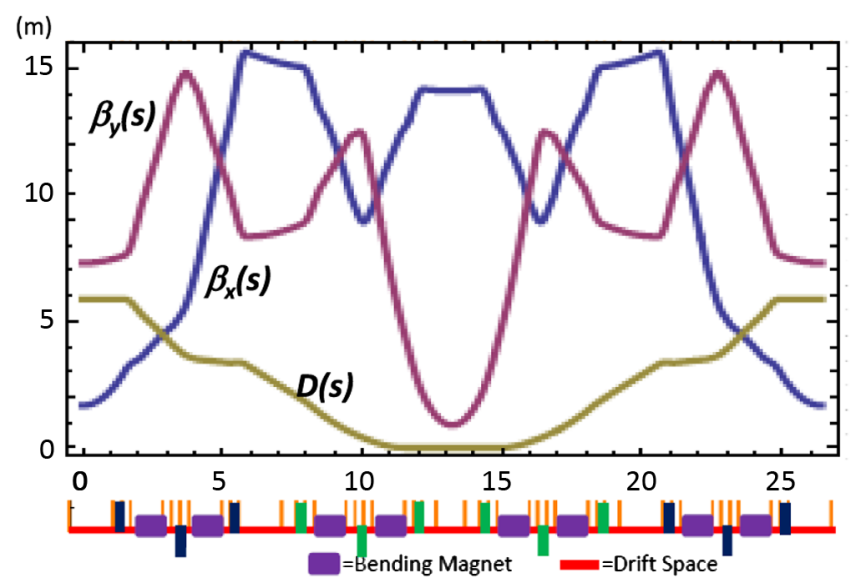

FIG. 7. Lattice functions and lattice for the single superperiodicity.
TABLE II. Parameters of the lattice.

\begin{tabular}{|c|c|c|c|c|c|}
\hline & $\begin{array}{l}\text { Length } \\
\text { (m) }\end{array}$ & $\begin{array}{c}\text { Bending } \\
\text { angle } \\
\text { (deg.) }\end{array}$ & $\begin{array}{l}\text { Edge } \\
\text { angle } \\
\text { (deg.) }\end{array}$ & $\begin{array}{c}\text { Curvature } \\
\text { (m) }\end{array}$ & $\begin{array}{c}\text { k-value } \\
\left(\mathrm{m}^{-2}\right)\end{array}$ \\
\hline D1 & 1.5 & & & & \\
\hline D2 & 0.3 & & & & \\
\hline D3 & 0.5 & & & & \\
\hline $\begin{array}{c}\text { D4 (space } \\
\text { between } \\
\text { QF2s) }\end{array}$ & 1.0 & & & & \\
\hline QF1 & 0.3 & & & & 0.75568 \\
\hline QD1 & 0.3 & & & & -0.53774 \\
\hline QF2 & 0.3 & & & & 0.40173 \\
\hline QD2 & 0.3 & & & & -0.8957 \\
\hline B & 1.1256 & 22.5 & 11.25 & 2.8662 & \\
\hline
\end{tabular}

where $E_{n}^{s}$ is the total energy of the synchronous particle of n-th turn, $Q$ is the charge state of an accelerating ion, $\eta$ is the slippage factor, and $\gamma_{s}$ is the relativistic $\gamma$ of the synchronous particle of n-th turn,

$$
\begin{aligned}
(\Delta E)_{n} & \equiv E_{n}-E_{n}^{s}, \quad \frac{\Delta p}{p}=\frac{(\Delta E)_{n}}{\left(\beta_{n}^{s}\right)^{2} E_{n}^{s}} \\
\eta & \equiv \frac{1}{\gamma_{T}^{2}}-\frac{1}{\gamma_{s}^{2}}
\end{aligned}
$$

The typical profiles of $V_{a c}$ and $V_{b b}$ are depicted in phase in Fig. 9. The rise and fall times of the voltage pulses are uniquely determined by the characteristics of the switching device and circuit parameters of the induction acceleration system, and they are fixed to be $35 \mathrm{~ns}$. Since these times are constant, the rising and falling period occupy relatively large spaces in phase near the end of acceleration.

Figure 10 shows the phase-space plot of macroparticles at different timings, where adiabatic damping in the momentum deviation is obvious. Some longitudinal emittance blowup associated with the reflection by the right barrier voltage is notable. The phenomena appear superimposed in

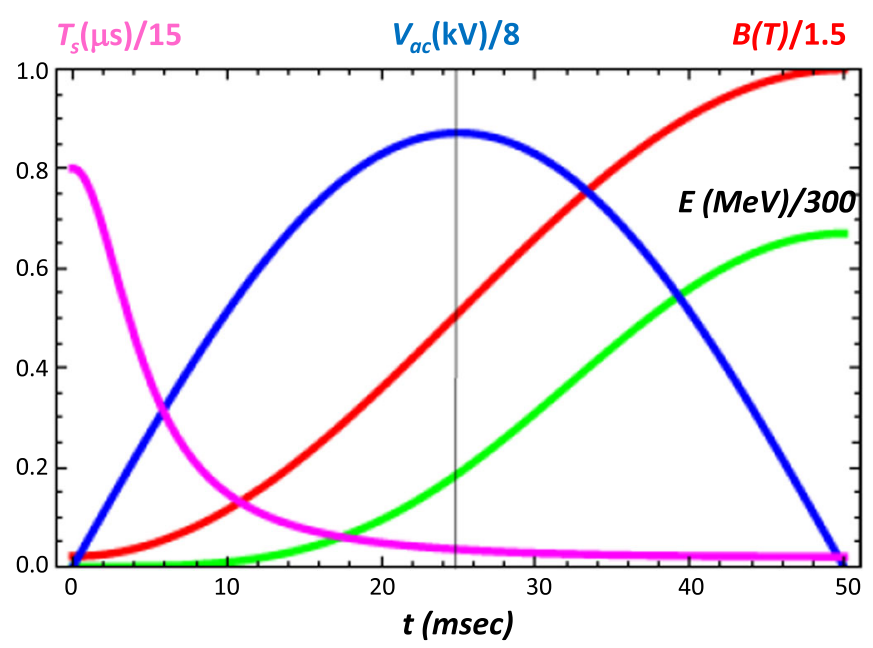

FIG. 8. Temporal evolutions of machine and beam parameters. 


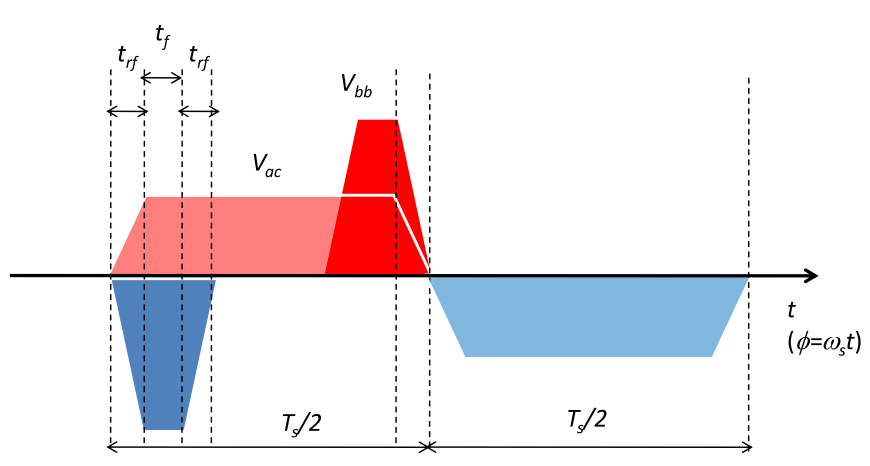

FIG. 9. $\quad V_{a c}$ and $V_{b b}$ profiles in time before extraction, where $T_{s}$ and $\omega_{s}$ are, respectively, the revolution time period and angular revolution frequency of the synchronous particle; $t_{r f}$ is the rising and falling time period of both voltage pulses (fixed to $35 \mathrm{~ns}$ ), and $t_{f}$ is the flat top time period, fixed to $T_{s} / 40$.

the phase space. The phase-space plot shown here is for the case of normal acceleration with no energy sweep extraction.

\section{B. Spill drop from the barrier bucket}

In the present simulation, the extraction of macroparticles starts near $25 \mathrm{~ms}$. For this purpose, the gate signals for the $V_{a c}$ voltage pulses are changed beyond the starting time of extraction so that the flat edge of $V_{a c}$ becomes equal to the rising bottom of positive $V_{b b}$. Particles entering into the positive barrier region, where the $V_{a c}$ voltage profile has a negative slope, are affected by an insufficient acceleration voltage, resulting in a larger negative momentum deviation. These particles leave the barrier bucket region or trapped region to move further downward. Eventually, they arrive at the boundary region of $\Delta p / p=-10^{-2}$, beyond which a particle entering into the electrostatic septum region is kicked inward in the horizontal direction by the electrostatic fields.

Typical examples of the phase plot with the $V_{a c}$ and $V_{b b}$ profiles are shown in Fig. 11. More of the fine structure of the phase-space plot is understood in Fig. 12. It can be seen that a small fraction of macroparticles continuously drifts down in the momentum space. At this stage, the spill is not controlled because no parameters have been optimized.

\section{Spill control}

The size of the spill must be controlled in time for the practical medical applications considered here. There are several methods that can be used to control the spill. Here, a
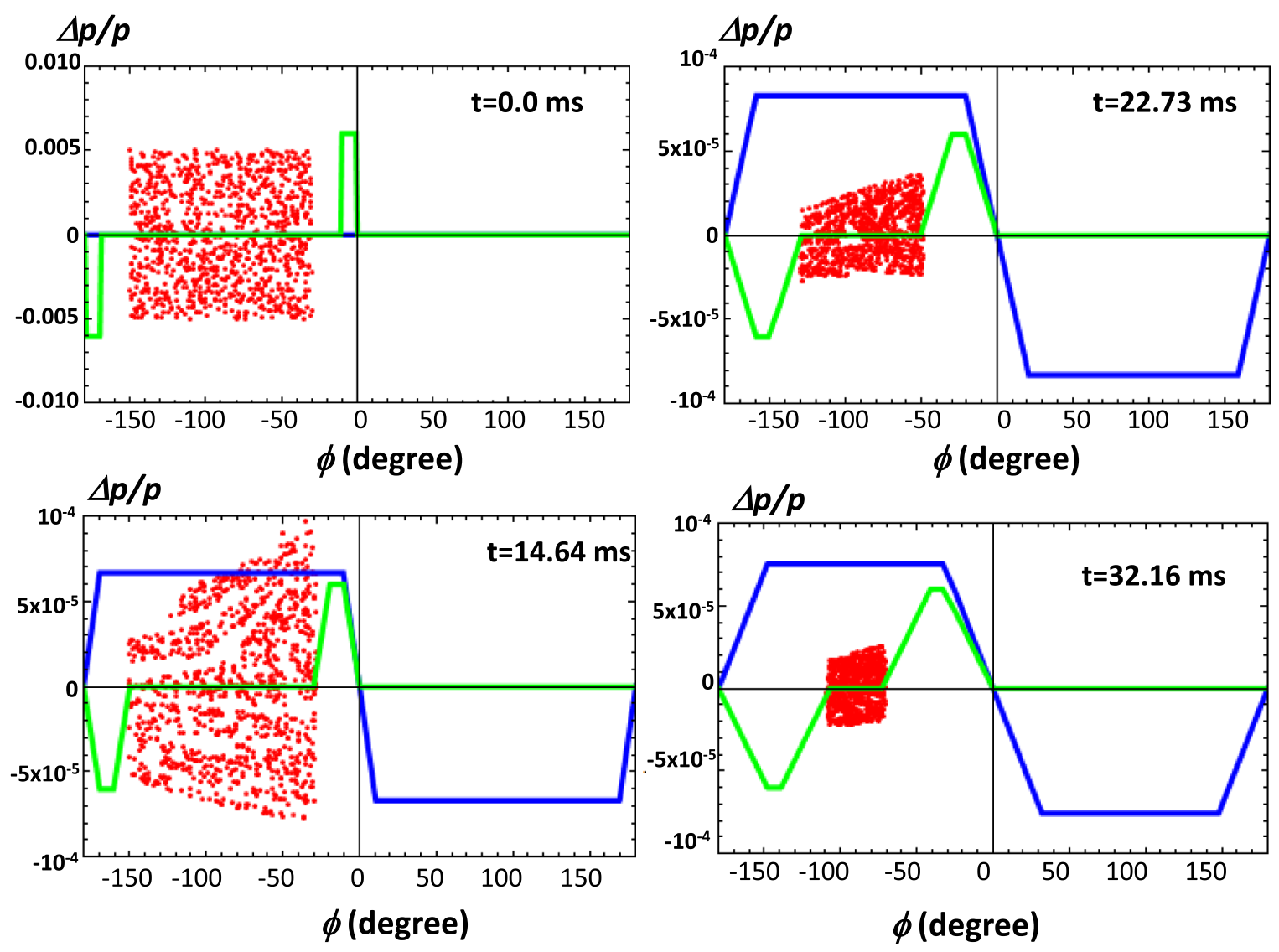

FIG. 10. Phase plots of tracked macroparticles in phase space with $V_{a c}$ and $V_{b b}$; voltage heights are shown in relative units. The asymmetric bunch profile seen in the early time period of acceleration is generated by a combination of the reflection due to the right hand barrier voltage pulse with a relatively steep voltage rising profile and dependence of the phase velocity on $\Delta p / p$. 
$\Delta p / p$
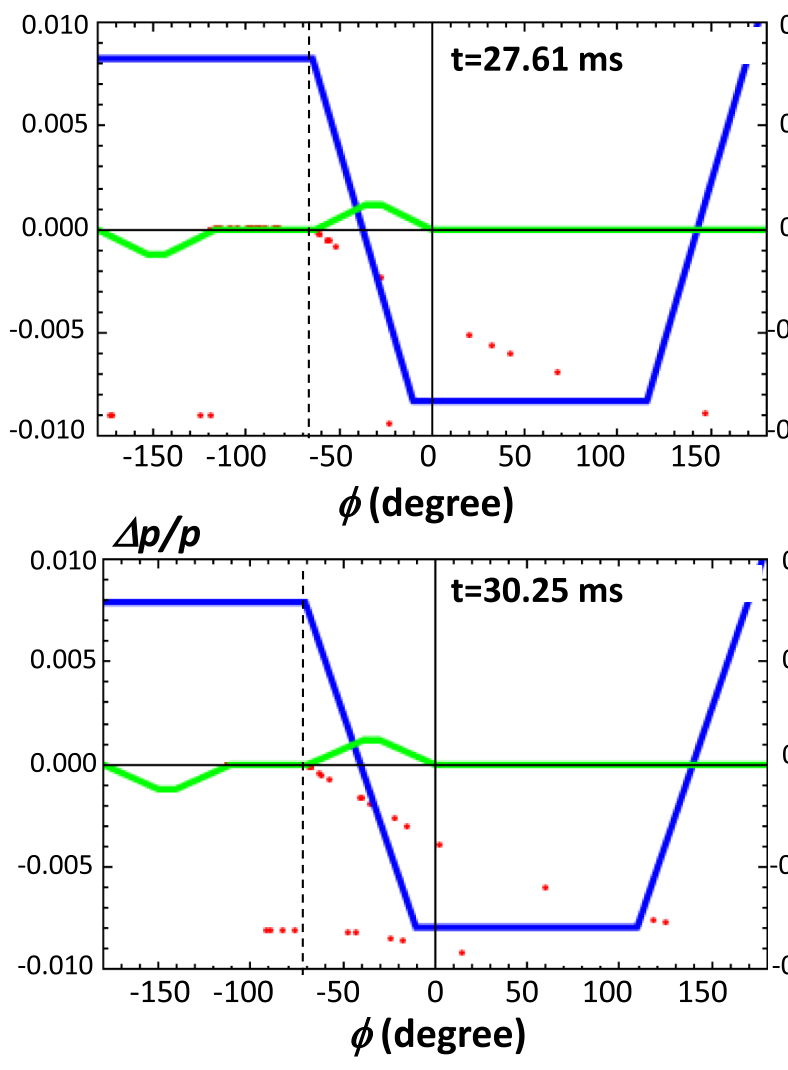

$\Delta p / p$

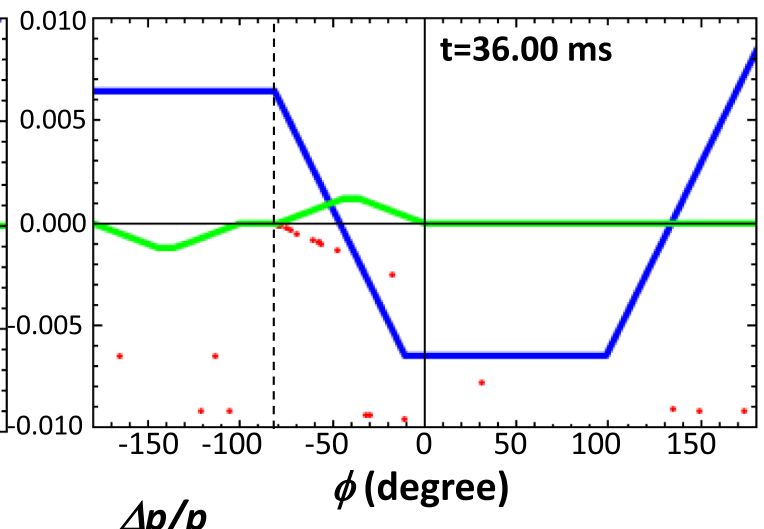

$\Delta p / p$

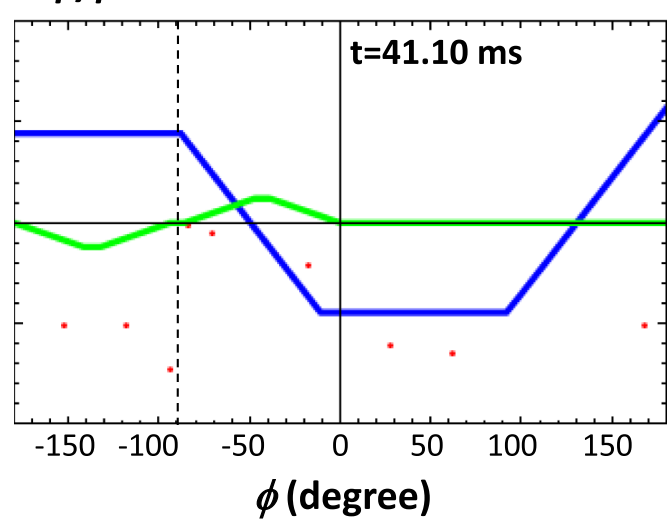

FIG. 11. Phase plots of macroparticles leaving the barrier bucket region, where the bunch core is invisible because its momentum spread is quite small. The positions of broken lines, from which particles leak, should be noted.

simple method, changing the turning-off time of $V_{a c}$ in a programmed manner, is described. The idea is quite simple. If some of the particles being trapped in the barrier bucket are not given the energy necessary to follow the guiding magnet pattern, they will leave the trapping region or the barrier bucket, drifting downward in the longitudinal phase space. This is a key feature of spill control. The acceleration is uniquely determined by $V_{a c}$. Its downhill profile in time is steep, and its starting phase for falling down in $V_{a c}$, which is indicated by $\phi_{\text {ext }}$ in Fig. 13, is manipulated by the gate control for the switching power supply. The timing for this is continuously adjusted in a programmed manner or by means of feedback from the spill monitoring system. The procedure for spill control is described below.

(1) $\phi_{\text {ext }}$ is instantaneously moved to near the left edge of the right barrier voltage pulse $\left(\phi_{b b}\right)$ at the start of spill extraction.

(2) Then, $\phi_{\text {ext }}$ is changed so as to satisfy the expected spill profile in a programmed manner or by monitoring the actually extracted spill profile.

For simplicity, the integrated spill profile is shown as a function of time since the start of extraction for three cases with different but constant values of $\phi_{\text {ext }}$. The simulations assumed 1,000 macroparticles. It is clear in Fig. 14 that the initial gradient of the integrated spill depends strongly on $\phi_{\text {ext }}$, although 1,000 macroparticles is insufficient for accuracy. As a demonstration, a typical case is given in Fig. 15, where the spill is kept to be almost constant during the extraction time period, from $25 \mathrm{~ms}$ to the end of acceleration.

An example with a constant spill profile in time, where the extraction is divided into three stages with different values of $\phi_{\text {ext }}$, is shown in Fig. 15. The linearity of the integrated spill in time is not sufficiently verified here. However, this result suggests that delicate control of $\phi_{\text {ext }}$ should allow realizing a desired spill profile.

\section{CRUCIAL DEVICES FOR ENERGY SWEEP EXTRACTION}

From the beam dynamics point of view, the most important issue is how an extracted beam with different energies in the acceleration cycle can be guided to the beam handling region in the transverse direction for 2D spot scanning along the extraction beam line. If all guiding devices, including the extraction devices, are ramped in the same way as the main guiding magnets in the ring, then the ideal orbit should be shared by all extraction timings and 


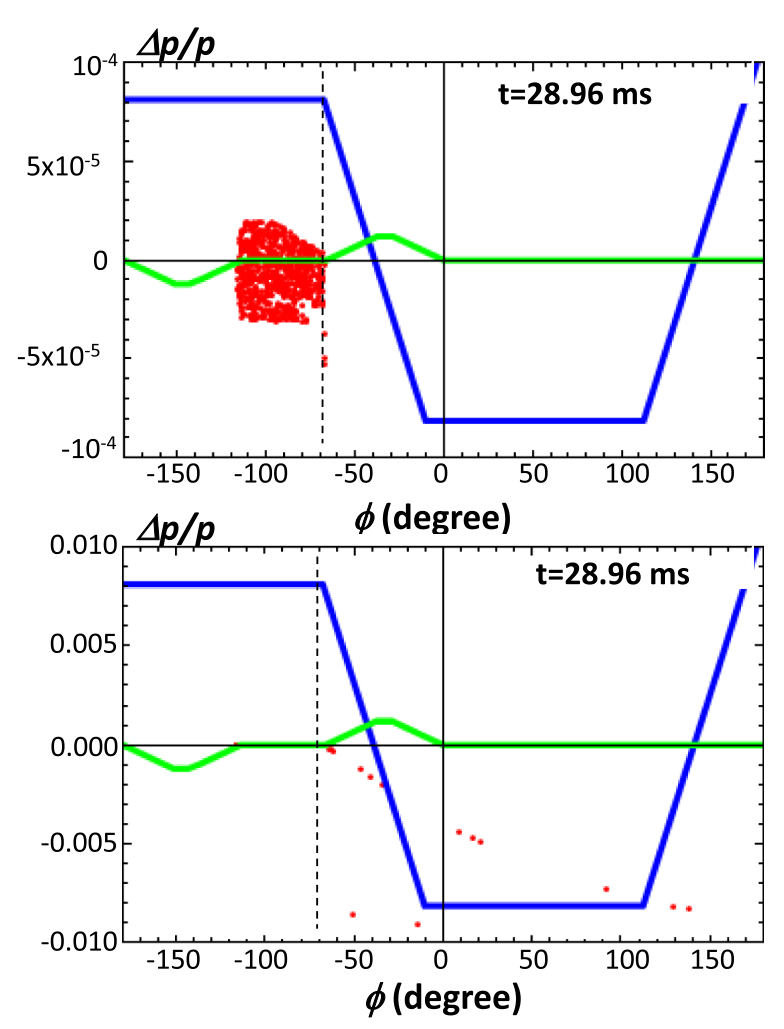

FIG. 12. More fine structure of the phase-space plot.

the extracted beams will have the same lattice function of the extraction beam line throughout the entire acceleration cycle. Extraction devices suitable for the present purpose, an electrostatic septum and a Lambertson magnet, which seem to be also practical from an engineering point of view, are considered here. The electrostatic septum is installed in the flat dispersion region, while the Lambertson magnet occupies the downstream straight section with the middlesized dispersion function, as explained in detail below.

\section{A. Electrostatic septum}

The maximum voltage generated by a DC power supply such as the Cockloft-Walton-type supply is

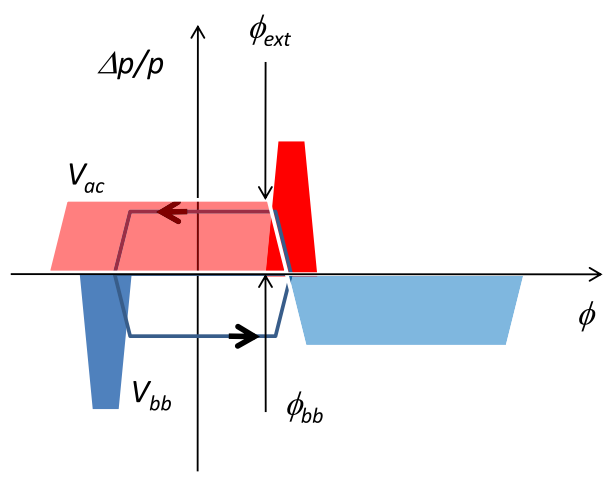

FIG. 13. Phase space with the $V_{a c}$ profile adjusted for extraction. usually added across the electrostatic septum gap as seen in Fig. 4. It seems difficult to introduce an additional pulse voltage generator in the electrostatic septum high-voltage circuit because the additional voltage pulse has to have a well-controlled time transient profile varying in a wide range of more than $50 \mathrm{kV}$, so as to correspond to the extraction from a middle stage in the acceleration cycle.

For this purpose, a novel charging method that employs a combination of high-power solid-state switches and a discharging switch has been studied. Its equivalent circuit is described in Fig. 16. Its nominal performance in operation is described below.

(1) The DC power supply $V_{1}$ continually charges the electrostatic septum through the charging resistor $\mathrm{R}_{1}$ to a voltage on the order of $-50 \mathrm{kV}$ before extraction.

(2) Switch 1 transfers the charge to the load from the other DC power supply $\mathrm{V}_{2}$ (having order $-120 \mathrm{kV}$ ) so that the required voltage profile $V(t)$, proportional to $\beta \gamma^{2}$, is satisfied beyond the extraction start time. The time constant of the voltage buildup is determined by the circuit constants, such as the resistance of the charging resistor $R_{2}$, the floating inductance $\mathrm{L}$, the parameters of the coaxial cable connecting $\mathrm{V}_{2}$ with the load, and the capacitance of the electrostatic septum. Switch 1 is discretely and repeatedly turned on and off in a well-programmed way.

(3) After completing the extraction, the discharging switch $S_{2}$ is turned on until the voltage of the load falls below $V_{1}$. The value of $R_{3}$ is the same as the characteristic impedance of the coaxial cable.

(4) Just after the acceleration cycle, the above process is repeated.

To confirm the feasibility of this programmed charging process, SPICE simulation was carried out, focusing particularly on the charging process from $V_{1}$ to $V_{2}$. An SI-Thyristor is assumed to be the solid-state switching element for $S_{1}$, which has rise and fall times of $200 \mathrm{~ns}$. Finite but relatively fast switching characteristics have no notable effects on the charging performance that are of present concern. The whole inductance in this circuit and the coaxial cable can be ignored for a time duration of millisecond order. Simulation results are shown in Fig. 17 where $\mathrm{R}_{1}$, $\mathrm{L}$, and $\mathrm{C}$ are assumed to be $9.58 \mathrm{M} \Omega, 1 \mu \mathrm{H}$, and $1200 \mathrm{pF}$, respectively. It is noted that the capacitance of $1200 \mathrm{pF}$ includes not only the capacitance of the load but also of the $10 \mathrm{~m}$ of coaxial cable. $\mathrm{S}_{1}$ is turned on and off 8 times during the assumed extraction time period, as shown in Fig. 18. It is noteworthy that the discrepancy from the ideal voltage profile is less than $1 \%$. Further improvement seems to be possible by introducing more frequent switching. There are other concerns, such as discharge in the load and mechanical stress on the septum wires (which is associated with the fast voltage change between $\mathrm{V}_{1}$ and $\mathrm{V}_{2}$ ). These should be studied so that they can be solved soon. 

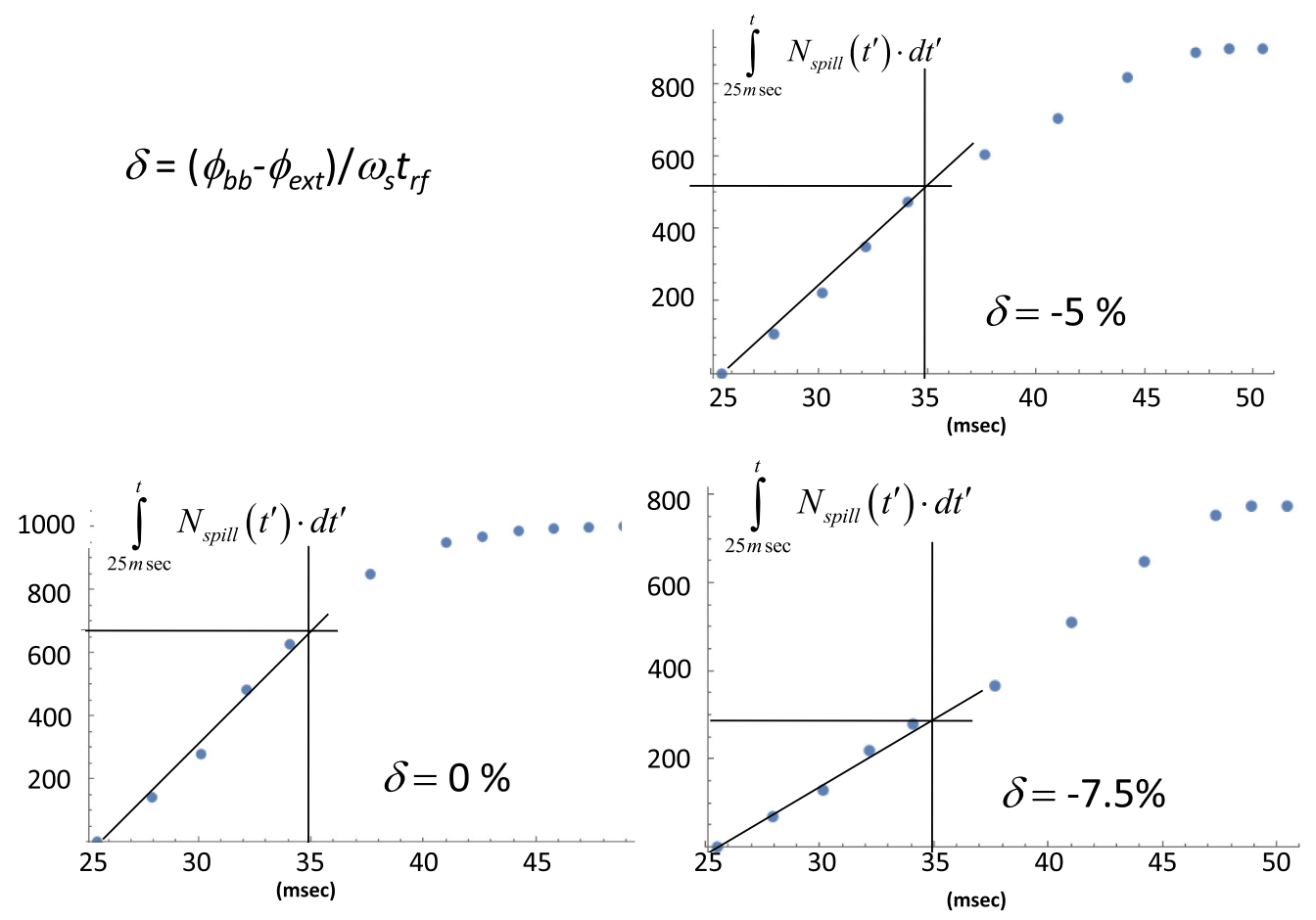

FIG. 14. Integrated spill for different values of $\delta$.

\section{B. Septum wire}

The beam loss associated with the energy sweep extraction is of significant concern. Some particles approaching the septum wire could hit the wire material before entering the high-voltage gap region during propagation though the septum region, where statistical and small-angle scattering and energy loss are inevitable. To minimize such effects, stranded carbon nanotube wire of $30-50 \mu \mathrm{m}$ diameter (Hitachi Zosen Corporation), which has recently become commercially available, is assumed. The mass density of such wire is lower than that of the usual carbon wire by a factor of 100 , leading to lower energy loss. Beam loss estimation is separately discussed in Sec. VI.

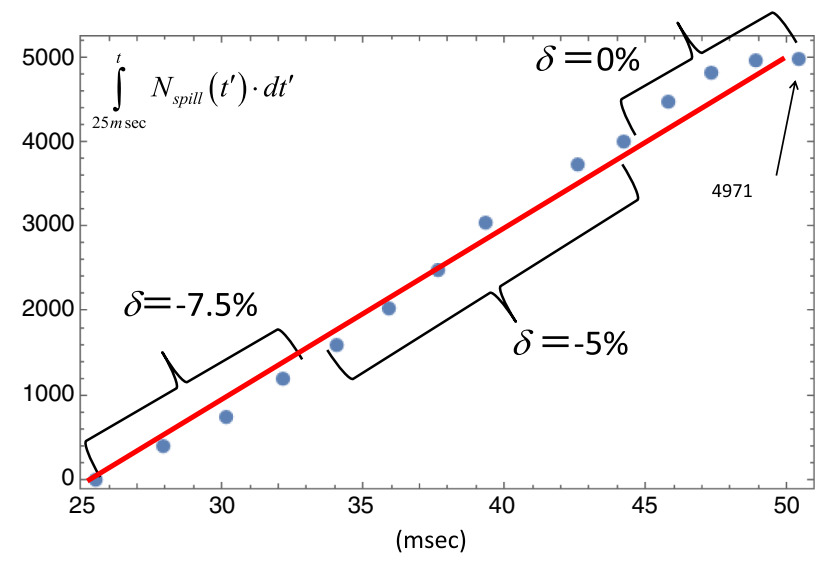

FIG. 15. Example of spill control for 5,000 macroparticles.

\section{Lambertson magnet}

A typical septum magnet is excited by a transient current, which here is generated in a circuit architecture consisting of a condenser bank, a resistor, a diode, and a thyristor as a switch, and a transformer (the magnet itself). The field profile is uniquely determined by the circuit parameters [11]. The peak field is used for extraction. Its time constant of change in the magnetic fields is either different from $10 \mathrm{~Hz}$ or does not meet the current demand. It is impossible to obtain the same current profile as that of the main magnet because Joule heat in the septum coil plate is too high to allow cooling it.

A laminated-core Lambertson magnet such as that shown in Fig. 19(a) is considered as an extraction magnet device. In this, it is emphasized that the Lambertson magnet is excited in a way similar to the excitation of the main magnets of the ring, and the cooling of the coil is not difficult. This is a crucial reason that this magnet is used in the present proposal. The Lambertson magnet extracts the beams in the vertical direction in a limited space.

The Lambertson magnet in the present extraction scheme is placed as shown in Fig. 19(b), which also schematically depicts the motion of an extracted particle in the betatron phase space. The angle and position of the beam line at the edge of the magnet still results in interference with the adjacent quadrupole magnet (QF2) and bending magnet, as shown as in Figs. 19(c) and 19(d). In such cases, the beam aperture for extraction must be put in the magnet return yoke. Perturbations from this modification on the circulating beam are limited and can be compensated. 


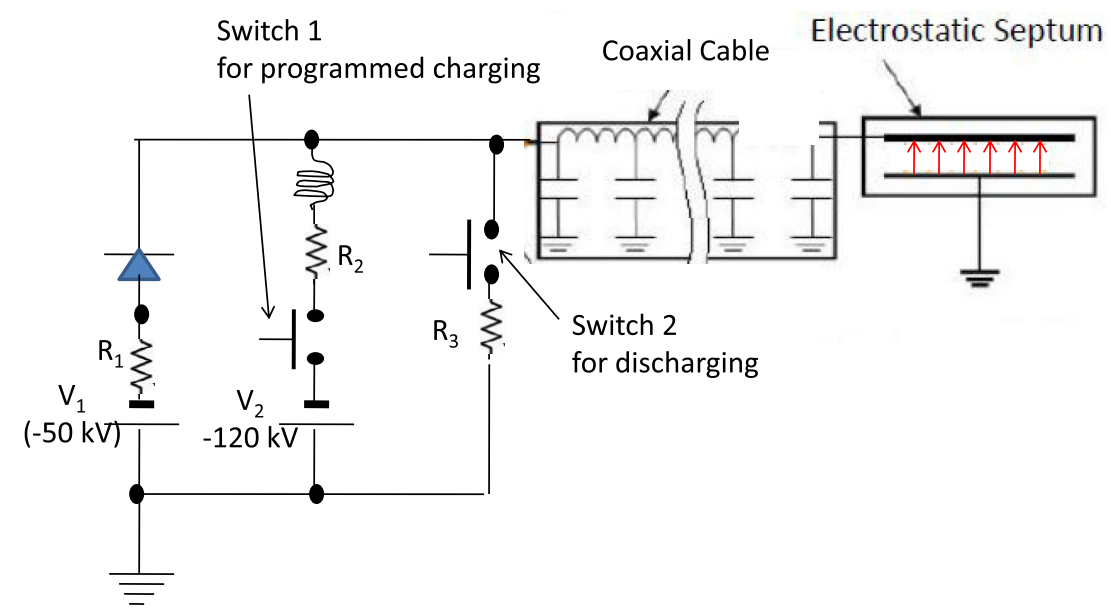

FIG. 16. Equivalent circuit of the electrostatic septum and its high voltage supplies.

Septum thickness of the Lambertson magnet is an important parameter. The necessary size can be evaluated from results of the spilled beam tracking simulation. Details are given in the next section.

\section{Momentum tracking guiding magnets}

All magnets must be ramped in a resonant circuit system that is the same as that in the ring magnets. Magnets, as many as possible, may be designed so as to be excited with the same current. It is obvious that grouping of magnets is

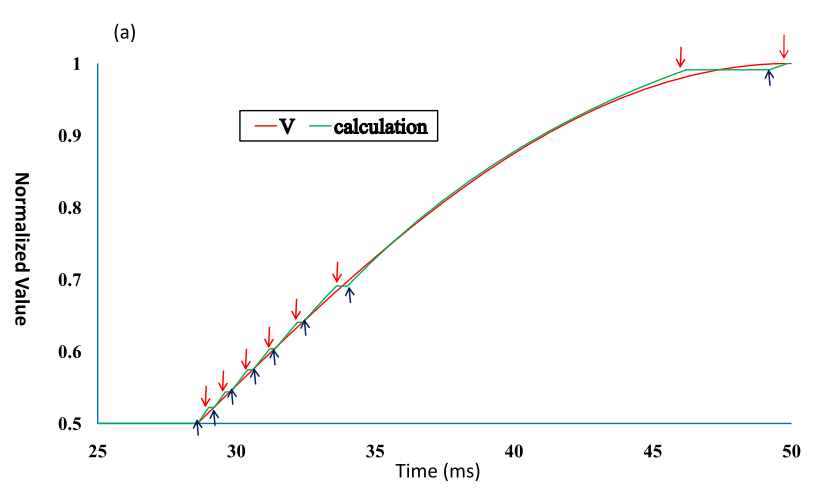

(b)

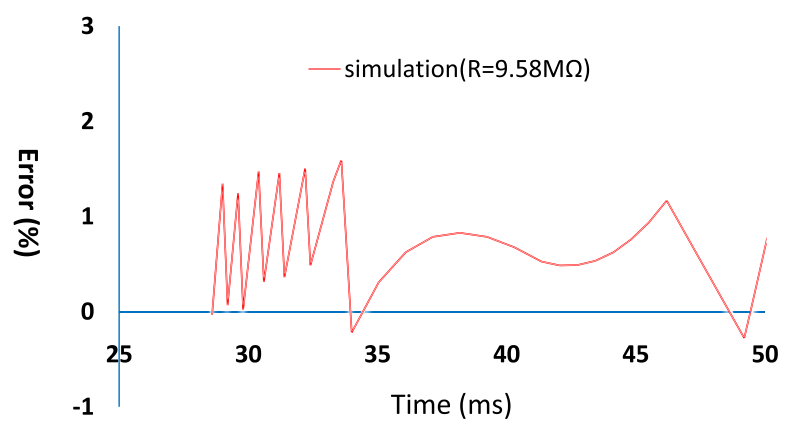

FIG. 17. (a) Ideal and simulated voltage patterns and (b) the discrepancy between both. useful for cost saving because it minimizes the number of resonant circuit systems.

\section{ESTIMATION OF BEAM SPILL QUALITY AND BEAM LOSS}

A particle spilled out of the barrier bucket should have a frozen transverse emittance that depends on its leaving time. For simplicity, an isolated spill is considered here, with the spill leaving the barrier bucket at once with fixed energy but varying horizontal emittance. The maximum emittance is the same as that of the beam core. Particles in the spill perform their own betatron oscillation depending on individual emittance. Associated with change in the momentum deviation, the spill gradually drifts toward the septum wire position. Particles on the outer edge of the spill that have the largest emittance begin to contact the septum wires. For now, assume that hitting the septum wire does not degrade the spill emittance and energy spread; this assumption will be justified later. The full beam size in the horizontal direction depends on the extraction time in the acceleration cycle. Assuming an average size of $1 \mathrm{~cm}$,

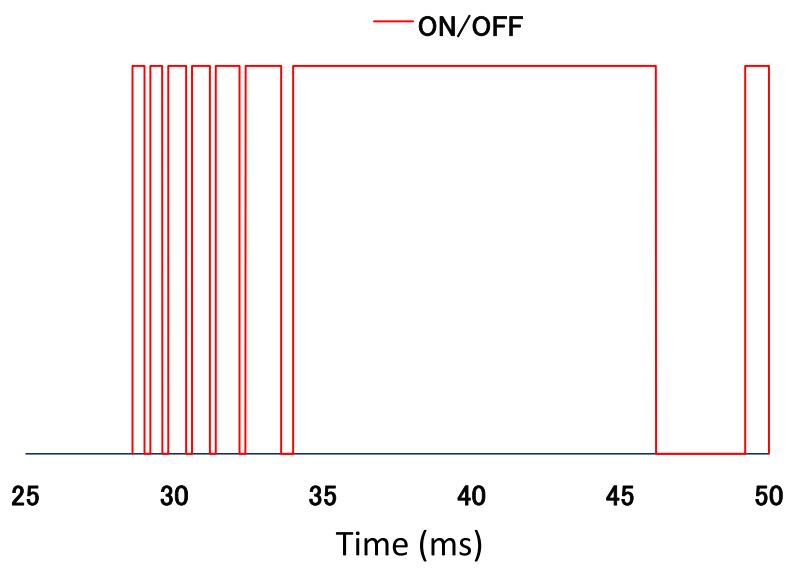

FIG. 18. Time table of Switch On/Off. 
(a)
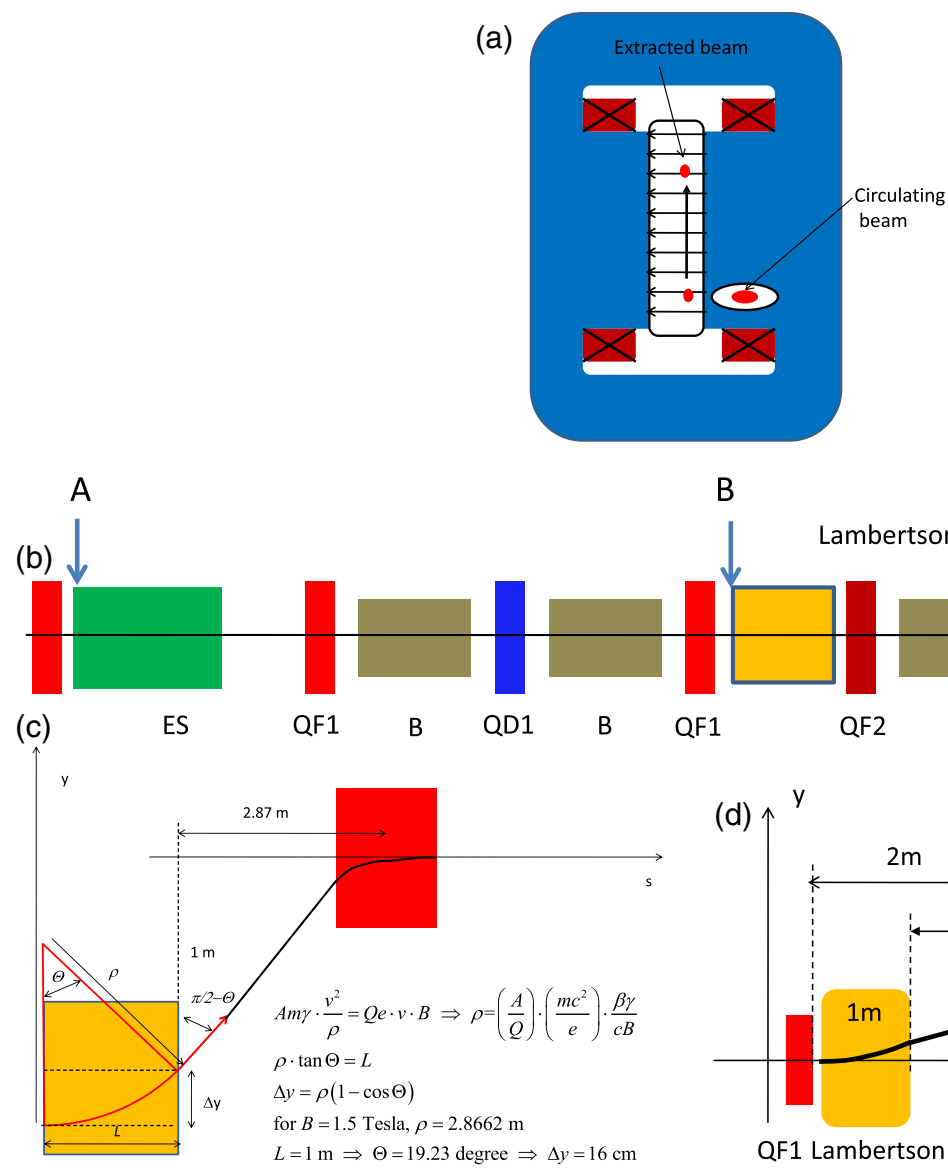

B

(b)

(c)

ES

$L=1 \mathrm{~m} \Rightarrow \Theta=19.23$ degree $\Rightarrow \Delta y=16 \mathrm{~cm}$

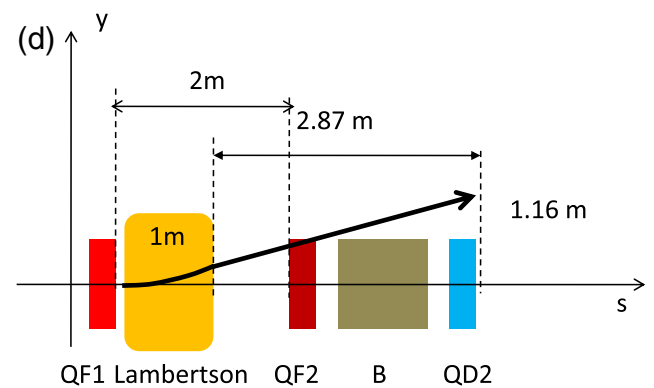

FIG. 19. (a) Cross-section of Lambertson magnet; (b) Schematic view of the extraction area with an electrostatic septum with voltage $120 \mathrm{kV}$, gap distance $3 \mathrm{~cm}$, and total length $2 \mathrm{~m} \mathrm{~A}$ : entrance of the septum region; B: entrance of the Lambertson magnet; (c) Extraction beam orbit and downstream; (d) Extraction orbit on the vertical plane.

the energy spread of a spilled beam is evaluated by dividing the full beam width by the dispersion function at the septum region, namely $1.7 \times 10^{-3}$. This is sufficiently small for the purpose of energy-sweeping irradiation.

As mentioned earlier, particles belonging to the same spill are not extracted in the same turn. The particles are extracted from largest emittance toward zero emittance across multiple turns, like peeling an apple. The spill is continuous in the present energy-sweep extraction method. The spilled beam observed at the entrance of the Lambertson magnet consists of particles with a momentum spread of $\Delta p / p=1.7 \times 10^{-3}$. The left-hand side of Fig. 20 shows a phase-space plot of an isolated beam spill just before extraction, which is monitored at the entrance of the septum region. The right-hand side of that figure shows a plot of extracted particles monitored at the entrance of the Lambertson magnet. These plots are obtained by 2D transformation of the deflected vector $(x, d x / d s)$ from the septum exit to the entrance of the Lambertson magnet. Considering the energy spread, the actual spilled beam should become as shown in the middle diagram of Fig. 20. Note that at the entrance of the Lambertson magnet, the dispersion function is $3 \mathrm{~m}$ and the beam size increases by a factor of about 3 because of increasing beta function. This beam spread has to be accommodated in the Lambertson aperture. Even considering this, a septum thickness of a few centimeters can be reserved. Field calculations confirm that the necessary fields are generated in the pole gap.

The average turn separation is larger than the size of the septum wire over almost the entire region of the extraction time period, excluding its last stage in Fig. 21. This means that the collision probability is far less than unity in this time period, and it is unnecessary to consider beam loss there because of the noninteger betatron tune, even considering betatron oscillation. Beyond $45 \mathrm{~ms}$, however, the narrow turn separation yields multiple passes through the septum wires for some fraction of spill with an extremely small emittance. Such a spill fraction is subject to energy loss and small-angle scattering at exit as a result of Rutherford scattering due to carbon atoms. Their magnitudes for a carbon nanowire material of $30 \mu \mathrm{m}(\delta)$ thickness are obtained as a function of projectile ion energy using the Monte-Carlo simulation tool SRIM (stopping and range of ions in matter) [12]. Figures 22a and 22b respectively show 


\section{A: at entrance of ES}

B: at entrance of Lambertson Magnet

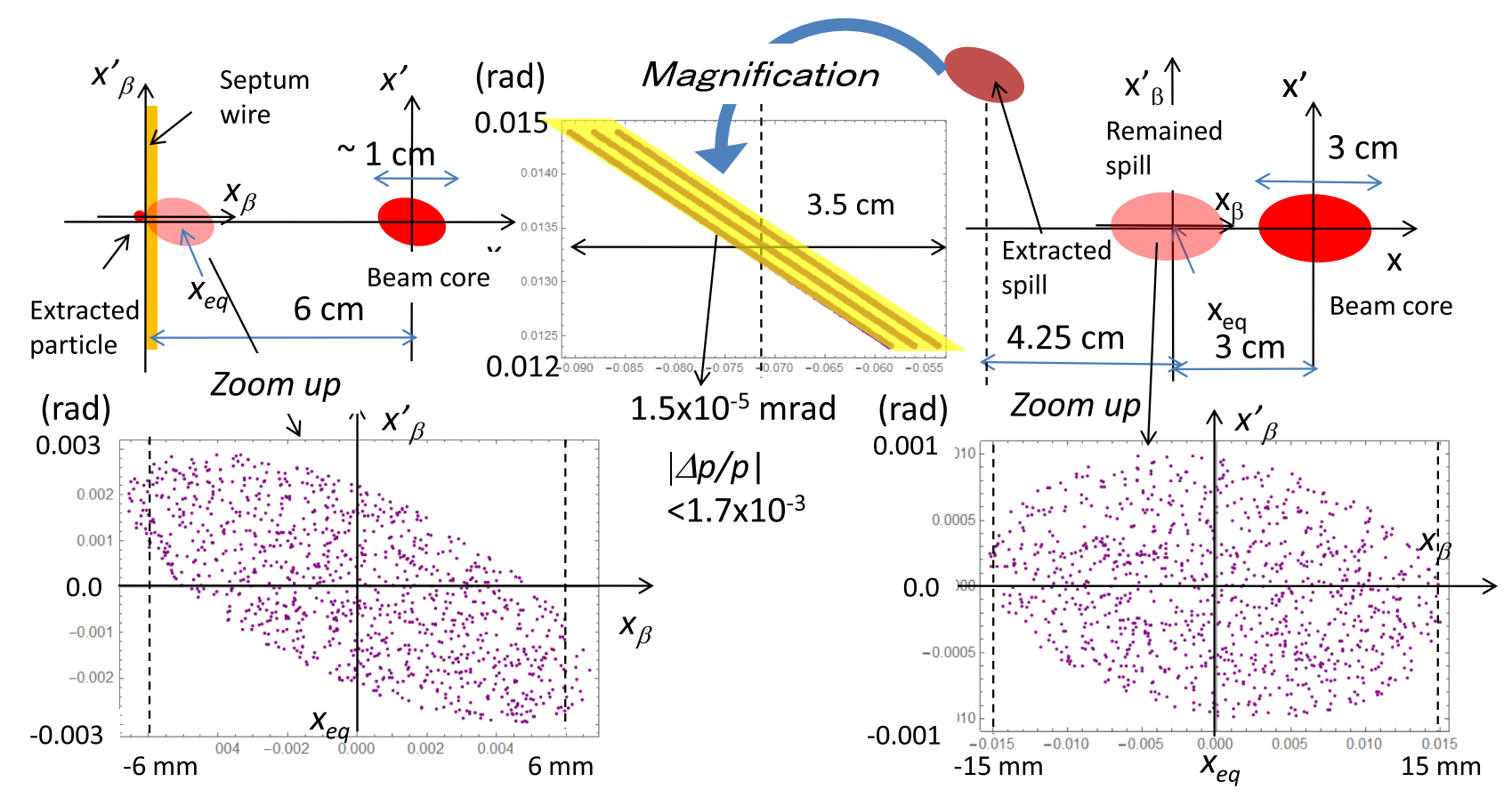

FIG. 20. Schematic phase plots of the circulating beam core, remaining spill, and spilled beam at the septum region and Lambertson magnet entrances, with tracking simulation results.

the energy loss and associated change in the momentum deviation. Here, the objective thickness is assumed to be $N \times \delta$, where $N$ is the total number of wires (200). The change in the momentum deviation is not small. This change seems to be sufficient to result in beam loss after the first collision.

The loss ratio is estimated by a simple geometrical model in the phase space shown in Fig. 23, where the dashed line represents the Courant-Snyder ellipse for a spill with an outer edge of $a$ (approx. $1 \mathrm{~cm}$ ). Simple algebra shows that for a particle placed in the inner Courant-Snyder ellipse, where $\beta_{x}(\mathrm{~s})=\beta * \approx 2 \mathrm{~m}$ and

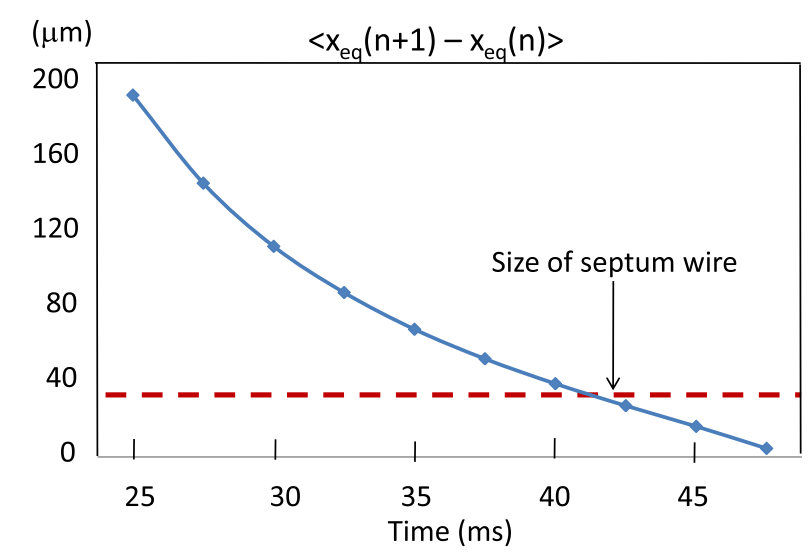

FIG. 21. Averaged turn separation vs. extraction timing. $\alpha_{x}(\mathrm{~s}) \approx 0$, its outer edge is half of the wire size $(\delta / 2)$, and so could penetrate the entire sequence of wires comprising $N$. The product of the gradient $\delta / 2 \beta *$ at Point $\mathrm{B}^{\prime}$ and the length of septum region of $L(2 \mathrm{~m})$ gives the excursion from the wire position, $L \delta / 2 \beta *$. Because $L / \beta * \approx 1$, we have an excursion of $\delta / 2$. Consequently, the upper/lower half of the ellipse naturally drifts to the positive/negative direction of $x$. Particles in the upper half-region are subject to excited coherent betatron motion in the subsequent turns. This process always takes place when the spill crosses the wires. The particles, which are located in the region represented by the rectangle in Fig. 23, experience the same phenomenon.

For simplicity, we define the beam loss rate as the ratio of the surface area $S_{\text {loss }}$ of the rectangular region to that of the outer ellipse $S_{\text {spill, }}$, assuming a uniform distribution in the transverse phase space, $r=S_{\text {loss }} / S_{\text {spill }}$. Substituting the parameter values into expression $r$, we have $2 \times 10^{-3}$. As mentioned earlier, this loss becomes significant with reduced turn separation. If we assume loss beyond $45 \mathrm{~ms}$ and uniform spill through the extraction, an overall loss rate of $4 \times 10^{-4}$ can be estimated. This magnitude seems to be tolerable.

Figure 24 shows the calculated results. The size is similar to $d x / d s$ at Point $\mathrm{B}^{\prime}$ over the entire acceleration time period. Emittance increase of a particle in the rectangular region in Fig. 23 due to the small angle scattering must also 


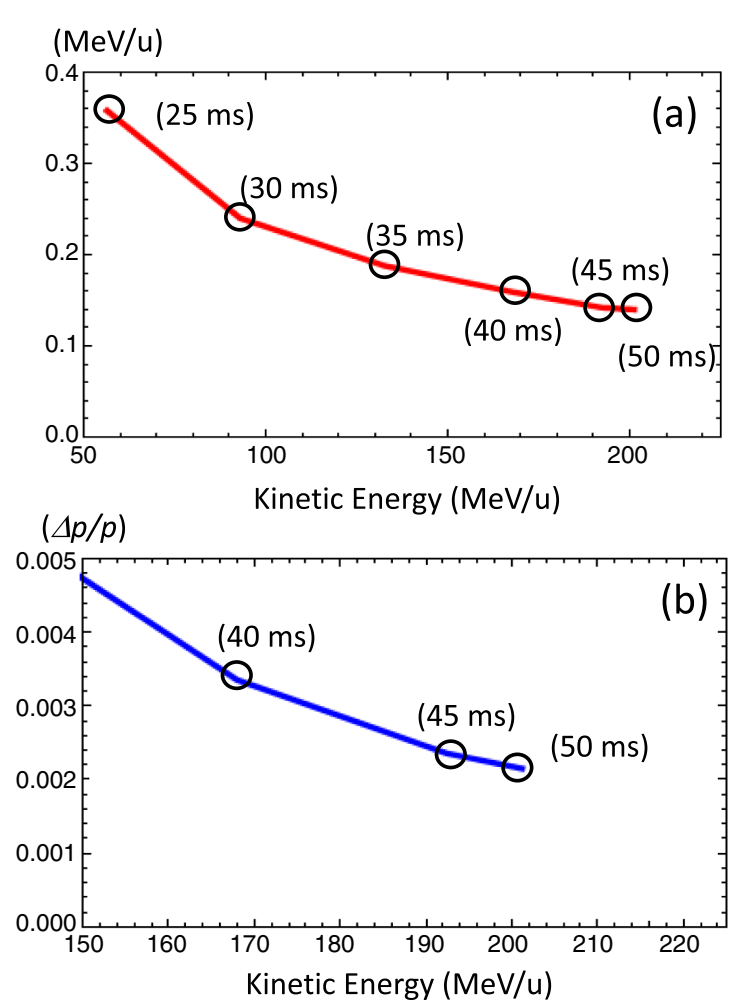

FIG. 22. (a) Energy loss vs. kinetic energy of a projectile carbon ion and (b) Momentum change per pass vs. kinetic energy of a projectile carbon ion.

be considered. However, we have already accounted for these particles as a candidate of loss.

The standard deviation of the scattering angle is obtained in the term of $\Delta x^{\prime}$ by code developed by Adachi, which is different by a factor of $60 \%$ from the value from SRIM. This difference is understood to result from differences in the definition of the scattering angle.

Further discussion concerning small-angle scattering and the resulting emittance increase and associated beam loss are beyond the scope of this paper.

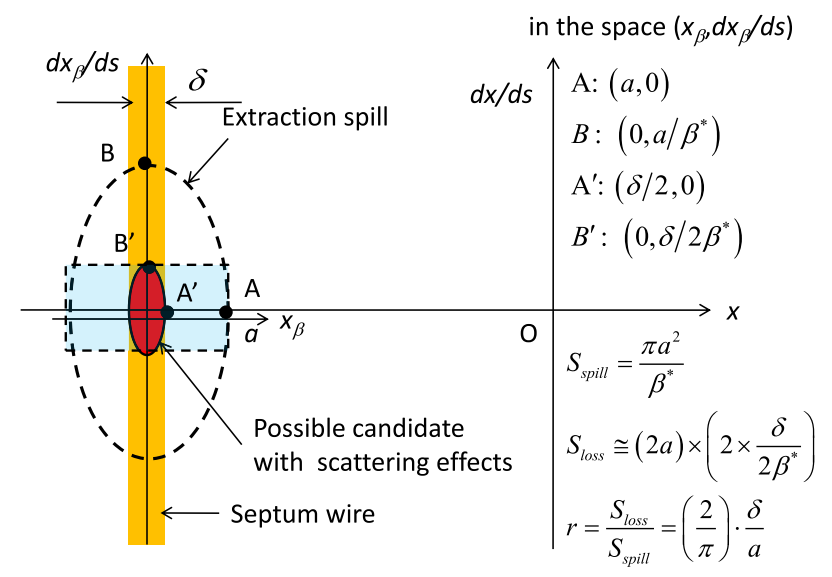

FIG. 23. Geometry near the septum wire for the extraction.

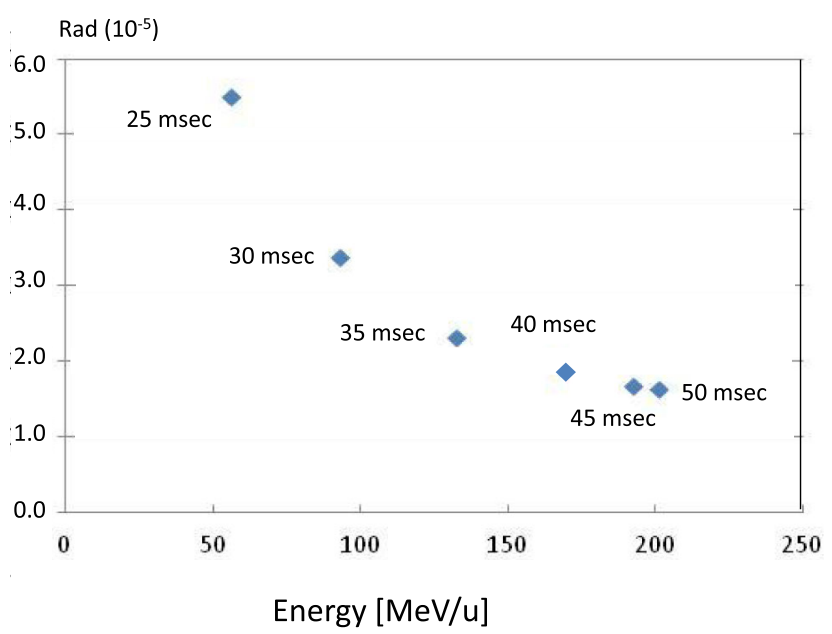

FIG. 24. RMS values for small scattering angle per wire.

\section{SUMMARY}

The function of energy sweep extraction in a fast-cycling induction synchrotron discussed here will allow spot scanning in depth on moving organs, which requires energy changes within a few tens of milliseconds. In a sense, the proposed technique seems to be an ideal method. However, this has not been realized yet. Details of the novel technique, which simply employs a combination of gatecontrolled induction acceleration and flexible reflection barrier voltage pulses, have been discussed. This method assumes a ring lattice optimized especially for the momentum dispersion function and an extraction system in which the electric and magnetic fields vary in time as expected. It is noted that the latter can be made possible by a novel electrostatic charging method that utilizes solid-state fast-switching elements. The development of such basic pulsed-power technology is quickly advancing.

The extraction scenario consisting of the electrostatic septum and Lambertson magnet seems to be the most promising. The separation from the circulating beam orbit and the remaining spill in the straight section for extraction may be enough to accommodate the septum thickness of the usual DC septum magnet. Also note that the DC septum magnet must be excited following the ramp pattern of the main magnet. We can propose other scenarios to avoid even the beam loss assumed here. The following approaches should be considered.

(1) Programmed energy sweep extraction is completed before $45 \mathrm{~ms}$. All particles are extracted before then.

(2) Programmed energy sweep extraction is completed before $45 \mathrm{~ms}$. Remaining particles are accelerated until $50 \mathrm{~ms}$, then extracted by a kicker system in just one turn.

Nevertheless, there are still several issues related to transverse beam emittance. The transverse spill beam emittance should depend on the extraction energy. It is necessary to study how the transverse spill emittance affects the 2D spot scanning, or in other words, what is 
required to avoid negative effects. These studies are left for future work. Here, a crucial idea has been simply discussed together with key technologies, which are inevitably needed to realize the idea.

\section{ACKNOWLEDGMENTS}

Discussions between one of the authors (K. T.) and J.P. Lidestri, who conducts the iRCMS project, at the Asian Forums for Accelerators and Detectors 2014 in Melbourne have strongly inspired the present study. We are indebted to Dr. Wake for field calculations of the Lambertson magnet. The present study has been partially supported by Grant-InAid for Scientific Research (B) (KAKENHI No. 15H03589).

[1] K. Hiramato, HITACHI proton therapy system with spot scanning, in Workshop on Hadron Beam Therapy of Cancer, 27th April, 2009, Erice, Italy, http://erice2009 .na.infn.it/TalkContributions/Hiramoto.pdf.

[2] J. P. Lidestri, S. G. Peggs, D. Trbojevic, and D. J. Brenner, iRCMS status and future plans, presentation at AFAD2014 (2014) https://accelerators.org.au/indico/ getFile.py/access? contribId $=57 \&$ sessionId $=31 \&$ res $I d=0 \&$ materialId $=$ slides $\&$ confId $=564$.

[3] K. Takayama, T. Yoshimoto, M. Barata, L. K. Wah, L. Xingguang, T. Iwashita, S. Harada, T. Adachi, T. Arai, D. Arakawa, H. Asao, E. Kadokura, T. Kawakubo, H. Nakanishi, Y. Okada, K. Okamura, K. Okazaki, A. Takagi, S. Takano, and M. Wake, Induction acceleration of heavy ions in the KEK digital accelerator: Demonstration of a fast-cycling induction synchrotron, Phys. Rev. ST Accel. Beams 17, 010101 (2014).C. M. Bhat, Applications of Barrier Bucket RF Systems at FERMILAB, Proceeding of International Workshop on Recent Progress in Induction Accelerators, pages 45-59, Tsukuba, Japan (KEK, 2006).

[4] N. J. Dougall, S. Bruggink, and K. P. Ebmeier, Systematic review of the diagnostic accuracy of $99 \mathrm{mTc}-\mathrm{HMPAO}$
SPECT in dementia, Am. J. Geriatr. Psychiatry 12, 554 (2004).

[5] K. Parodi, W. Enghardt, and Haberer, In-beam PET measurements of $\beta^{+}$radioactivity induced by proton beams, Phys. Med. Biol. 47, 21 (2002); P. Crespo, G. Shakrin, and W. Enghardt, On the detector arrangement for in-beam PET for hadron therapy monitoring, Phys. Med. Biol. 51, 2143 (2006); G. Sportelli, N. Belcari, N. Camarlinghi, G. A. P. Cirrone, G. Cuttone, S. Feretti, A. Krran, J. E. Ortuno, F. Romano, and A. Santos, First fullbeam PET acquisitions in proton therapy with a modular dual-head dedicated system, Phys. Med. Biol. 59, 43 (2013).

[6] Summary Report, Workshop on Ion Beam Therapy (2013) http://science.energy.gov/ /media/hep/pdf/accelerator-rdstewardship/Workshop_on_Ion_Beam_Therapy_Report_ Final_R1.pd.

[7] K. Okamura, Y. Yamada, M. Wake, and K. Takayama, Development of high power and law inductance SiC-JFET power module for high repetition rate pulsed power, 5th Euro-Asian Pulsed Power Conference (Kumamoto, Japan OB1-1, 2014).

[8] Proton-Ion Medical Machine Study (PIMMS), CERN/PS 2000-007 (DR) (2000), https://cdsweb.cern.ch/record/ 449577/files/ps-2000-007.pdf.

[9] N. Munemoto, K. Takayama, S. Takano, M. Okamura, and M. Kumaki, Development of the $C^{6+}$ laser ablation ion source for the KEK digital accelerator, Rev. Sci. Instrum. 85, 02B922 (2014).

[10] H. Kobayashi, T. Kawakubo, and A. Tokuchi, Electrostatic injection kicker for the KEK digital accelerator driven by SI-thyristor matrix array power system, 5th Euro-Asian Pulsed Power Conference (Kumamoto, Japan OB1-2, 2014).

[11] T. Kawakubo, Injection/Extraction, Lecture at KEK-Nuclear Malaysia-Sokendai Accelerator School in Nuclear Malaysia, 2014.

[12] J. F. Ziegler, M. Ziegler, and J. Biersack, SRIM - The stopping and range of ions in matter, Nucl. Instrum. Methods Phys. Res., Sect. B 268, 1818 (2010). 